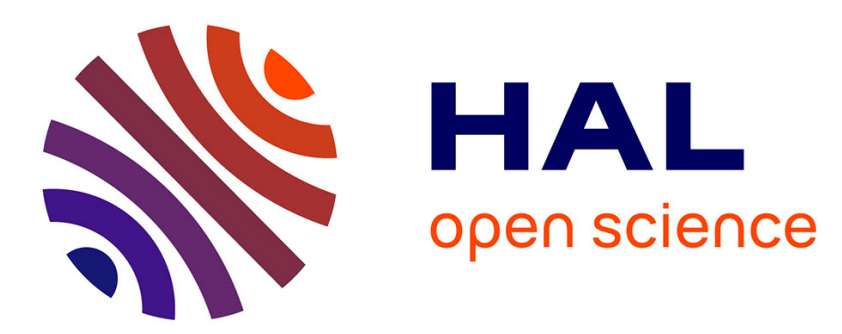

\title{
On the capillary motion of arbitrary clusters of spherical bubbles. Part 1. General theory
}

Antoine Sellier

\section{To cite this version:}

Antoine Sellier. On the capillary motion of arbitrary clusters of spherical bubbles. Part 1. General theory. Journal of Fluid Mechanics, 2004, april (504), pp.391-401. 10.1017/s0022112004008146. hal-01024957

\section{HAL Id: hal-01024957 \\ https://hal-polytechnique.archives-ouvertes.fr/hal-01024957}

Submitted on 29 Jul 2014

HAL is a multi-disciplinary open access archive for the deposit and dissemination of scientific research documents, whether they are published or not. The documents may come from teaching and research institutions in France or abroad, or from public or private research centers.
L'archive ouverte pluridisciplinaire HAL, est destinée au dépôt et à la diffusion de documents scientifiques de niveau recherche, publiés ou non, émanant des établissements d'enseignement et de recherche français ou étrangers, des laboratoires publics ou privés. 


\title{
On the capillary motion of arbitrary clusters of spherical bubbles. Part 1. General theory
}

\author{
By A. SELLIER \\ LadHyX, Ecole Polytechnique, 91128 Palaiseau Cédex, France
}

(Received 21 October 2003 and in revised form 17 January 2004)

A new and systematic approach is proposed to determine the migration of torque-free spherical bubbles immersed in a steady and non-uniform unbounded Stokes flow and subject to arbitrary capillary effects. The advocated procedure appeals to only a very few quantities on the surface of each bubble and is therefore suitable for a future numerical treatment of arbitrary clusters of bubbles. For a single bubble, the theory allows a straightforward analytical implementation and the predicted results agree well with Hetsroni \& Haber (1970), Hetsroni et al. (1971) and Subramanian (1985). The thermocapillary motion of non-conducting spherical bubbles freely suspended in a quiescent liquid in the presence of an arbitrary ambient temperature $T_{\infty}$ is considered and it is shown that it is futile to determine the disturbed temperature field, whatever $T_{\infty}$, once bubbles are equivalent (i.e. experience the same velocity in a given uniform temperature gradient $\nabla T_{\infty}$, as obtained by Acrivos et al. 1990 and Wang et al. 1994).

\section{Introduction}

As shown both experimentally and theoretically by Young, Goldstein \& Block (1959) for vanishing Reynolds and Marangoni numbers, a small spherical bubble freely suspended in an unbounded and quiescent Newtonian liquid of viscosity $\mu$ migrates when its surface tension $\gamma$ is non-uniform. For instance, if $\gamma$ depends on temperature $T$ with $\mathrm{d} \gamma / \mathrm{d} T$ uniform the so-called thermocapillary velocity $\boldsymbol{U}$ of a single and non-conducting bubble of radius $a$ subject to the uniform temperature gradient $\nabla T_{\infty}$ is

$$
\boldsymbol{U}=-\frac{a}{2 \mu} \frac{\mathrm{d} \gamma}{\mathrm{d} T} \nabla T_{\infty} .
$$

In practical applications clusters of bubbles occur and, if equivalent bubbles (having the same velocity (1.1) when isolated) do not interact (Acrivos, Jeffrey \& Saville 1990; Wang, Maury \& Acrivos 1994), particle-particle interactions in general strongly affect the zeroth-order solution (1.1) for arbitrary bubbles. These interactions have been thoroughly addressed for two-bubble clusters by using spherical bipolar coordinates (Meyyappan, Wilcox \& Subramanian 1983; Feuillebois 1989; Keh \& Chen 1990), or the method of reflections (Anderson 1985; Meyyappan \& Subramanian 1984; Sun \& Hu 2002) and twin multipole expansions (Satrape 1992; Wang et al. 1994). Unfortunately, for more than two non-equivalent bubbles available results are restricted to three-bubble chains (Keh \& Chen 1992, 1993; Wei \& Subramanian 1993) and the general treatment advocated in Keh \& Chen (1993) becomes so unwieldy for fully three-dimensional configurations (even for three bubbles) that it was practically impossible to deal with more realistic geometries. This paper introduces a new approach valid for arbitrary capillary effects and clusters of bubbles which will 

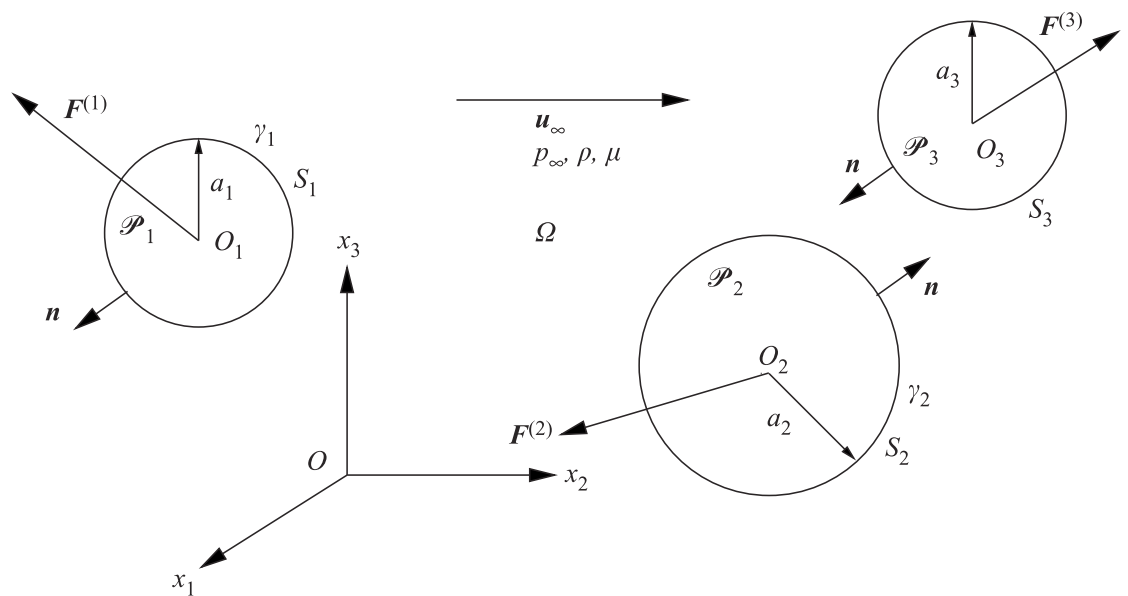

Figure 1. A fully three-dimensional $N$-bubble cluster subject to arbitrary ambient steady

Stokes flow $\left(\boldsymbol{u}_{\infty}, p_{\infty}\right)$, capillary effects $\left(\gamma_{1}, \ldots, \gamma_{N}\right)$ and net forces $\boldsymbol{F}^{(n)}$ (case of $N=3$ ).

not result in an increasing complexity in implementation as the number of bubbles increases.

\section{A general theory for arbitrary capillary effects}

Throughout this section torque-free but not necessarily force-free bubbles experiencing arbitrary capillary effects are treated.

\subsection{Assumptions and governing equations}

We consider, as sketched in figure $1, N \geqslant 1$ torque-free spherical bubble(s) $\mathscr{P}_{n}$ immersed in an unbounded Newtonian liquid of uniform density $\rho$ and viscosity $\mu$ and subject to a non-uniform steady Stokes $\left(\boldsymbol{u}_{\infty}, p_{\infty}\right)$ and arbitrary capillary effects. The resulting non-uniform surface tension $\gamma_{n}$ on the boundary $S_{n}$ of $\mathscr{P}_{n}$ is assumed to be high enough to keep $\mathscr{P}_{n}$ spherical with centre $O_{n}$, radius $a_{n}$ and unit outward normal $\boldsymbol{n}$. Since it is also defined inside each bubble the ambient Stokes flow $\left(\boldsymbol{u}_{\infty}, p_{\infty}\right)$ with stress tensor $\sigma_{\infty}$ applies zero net force and torque on $\mathscr{P}_{n}$. Thus, denoting by $M$ a point on $S_{n}$, one obtains

$$
\int_{S_{n}} \boldsymbol{\sigma}_{\infty} \cdot \boldsymbol{n} \mathrm{d} S_{n}=\mathbf{0}, \quad \int_{S_{n}} \boldsymbol{O}_{n} \boldsymbol{M} \wedge \boldsymbol{\sigma}_{\infty} \cdot \boldsymbol{n} \mathrm{d} S_{n}=\mathbf{0} .
$$

In the fluid domain $\Omega$, the liquid has pressure $p_{\infty}+p$ and velocity $\boldsymbol{u}_{\infty}+\boldsymbol{u}$ of scale $U$, the Reynolds number $R e=\rho U a / \mu$ with $a=\max \left(a_{n}\right)$ is sufficiently small to neglect all inertial effects and the migration of bubbles is assumed quasi-static. Accordingly, the perturbation flow $(\boldsymbol{u}, p)$ obeys the steady Stokes equations and the far-field behaviour

$$
\mu \nabla^{2} \boldsymbol{u}=\nabla p \quad \text { and } \quad \nabla \cdot \boldsymbol{u}=0 \quad \text { in } \quad \Omega,(\boldsymbol{u}, p) \rightarrow(\mathbf{0}, 0) \text { at } \infty .
$$

For spherical bubbles we ignore the normal stress balance on each surface $S_{n}$ (Satrape 1992). If $\boldsymbol{\sigma}$ denotes the stress tensor induced by $(\boldsymbol{u}, p)$ the boundary conditions then are

$$
\begin{aligned}
& \boldsymbol{u} \cdot \boldsymbol{n}=\left[\boldsymbol{U}^{(n)}-\boldsymbol{u}_{\infty}\right] \cdot \boldsymbol{n} \quad \text { and } \quad \boldsymbol{\sigma} \cdot \boldsymbol{n}-[\boldsymbol{n} \cdot \boldsymbol{\sigma} \cdot \boldsymbol{n}] \boldsymbol{n} \\
& =-\nabla_{s} \gamma_{n}-\boldsymbol{\sigma}_{\infty} \cdot \boldsymbol{n}+\left[\boldsymbol{n} \cdot \boldsymbol{\sigma}_{\infty} \cdot \boldsymbol{n}\right] \boldsymbol{n} \text { on } S_{n}
\end{aligned}
$$


where $\nabla_{s}$ designates the gradient along the surface $S_{n}$ of the torque-free bubble $\mathscr{P}_{n}$ which translates only at the unknown velocity $\boldsymbol{U}^{(n)}$ (the surface $S_{n}$ does not rotate but the inviscid gas inside may experience its own motion). Owing to the property $\boldsymbol{O}_{n} \boldsymbol{M} \wedge \boldsymbol{n}=\mathbf{0}$, the second condition (2.3) and Appendix A it is clear that

$$
\int_{S_{n}} \boldsymbol{O}_{n} \boldsymbol{M} \wedge\left[\left(\boldsymbol{\sigma}_{\infty}+\boldsymbol{\sigma}\right) \cdot \boldsymbol{n}\right] \mathrm{d} S_{n}=\int_{S_{n}} \nabla_{s} \gamma_{n} \wedge \boldsymbol{O}_{n} \boldsymbol{M} \mathrm{d} S_{n}=\mathbf{0}
$$

and the disturbed flow $\left(\boldsymbol{u}_{\infty}+\boldsymbol{u}, p_{\infty}+p\right)$ indeed applies a zero net torque on each bubble $\mathscr{P}_{n}$ (with respect to its centre $O_{n}$ ). Recalling (2.1), we thus supplement (2.2)(2.3) for bubbles of prescribed net force $\boldsymbol{F}^{(n)}$ (such as buoyancy) with the following conditions:

$$
\boldsymbol{F}^{(n)}=-\int_{S_{n}} \boldsymbol{\sigma} \cdot \boldsymbol{n} \mathrm{d} S_{n}
$$

For arbitrary $N$-bubble clusters, ambient Stokes flow $\left(\boldsymbol{u}_{\infty}, p_{\infty}\right)$, net forces $\boldsymbol{F}^{(n)}$ and capillary effects $\left(\gamma_{1}, \ldots, \gamma_{N}\right)$ the generalized velocity $\boldsymbol{X}=\left(\boldsymbol{U}^{(1)}, \ldots, \boldsymbol{U}^{(N)}\right)$ is obtained by solving (2.2)-(2.3) in conjunction with (2.5), a very challenging task. A conceivable numerical strategy may consist of first devising an algorithm that approximates in the whole unbounded fluid domain the solution to (2.2)-(2.3) and the resulting net forces on the bubbles for any given entry $\boldsymbol{X}$. Starting with a suitable guess value $\boldsymbol{X}_{g}$ this Code is then used in an iterative fashion to enforce the conditions (2.5). Unfortunately, even when resorting for instance to a finite element technique this approach will become prohibitively involved and CPU time consuming for fully three-dimensional configurations because the liquid domain $\Omega$ is three-dimensional and unbounded. As shown in $\S 2.2$, it is however possible to judiciously select $3 N$ Stokes flows that permit us to obtain $\boldsymbol{X}$ by evaluating only surface quantities on the entire two-dimensional boundary $S=\cup_{n=1}^{N} S_{n}$.

\subsection{Advocated approach}

Henceforth, we adopt Cartesian coordinates $\left(O, x_{1}, x_{2}, x_{3}\right)$ with $\boldsymbol{x}=\boldsymbol{O} M, x_{i}=\boldsymbol{x} \cdot \boldsymbol{e}_{i}$ and $r=|\boldsymbol{x}|$. For $i \in\{1,2,3\}$ and $n \in\{1, \ldots, N\}$ let us introduce the Stokes flow $\left(\boldsymbol{u}_{i}^{(n)}, p_{i}^{(n)}\right)$ with stress tensor $\boldsymbol{\sigma}_{i}^{(n)}$ such that

$\mu \nabla^{2} \boldsymbol{u}_{i}^{(n)}=\nabla p_{i}^{(n)} \quad$ and $\quad \nabla \cdot \boldsymbol{u}_{i}^{(n)}=0 \quad$ in $\quad \Omega,\left(\boldsymbol{u}_{i}^{(n)}, p_{i}^{(n)}\right) \rightarrow(\mathbf{0}, 0) \quad$ as $\quad r \rightarrow \infty$,

$\boldsymbol{u}_{i}^{(n)} \cdot \boldsymbol{n}=\delta_{n m} \boldsymbol{e}_{i} \cdot \boldsymbol{n} \quad$ and $\quad \boldsymbol{\sigma}_{i}^{(n)} \cdot \boldsymbol{n}-\left[\boldsymbol{n} \cdot \boldsymbol{\sigma}_{i}^{(n)} \cdot \boldsymbol{n}\right] \boldsymbol{n}=\mathbf{0} \quad$ on $\quad S_{m} \quad$ for $\quad m=1, \ldots, N$,

with $\delta$ the Kronecker symbol. For this flow the bubble $\mathscr{P}_{n}$ has velocity $\boldsymbol{e}_{i}$, other bubbles are motionless and there is neither ambient flow nor capillary effects. The surface traction $\boldsymbol{\sigma}_{i}^{(n)} \cdot \boldsymbol{n}$ is normal on each boundary $S_{m}$ and in general applies a nonzero net force on $\mathscr{P}_{m}$. Moreover, $\left(\boldsymbol{u}_{i}^{(n)}, p_{i}^{(n)}\right)$ permits us to express for $(\boldsymbol{u}, p)$ satisfying (2.2)-(2.3) the integral

$$
I_{i}^{(n)}=\int_{S_{n}}\left(\boldsymbol{e}_{i} \cdot \boldsymbol{n}\right)(\boldsymbol{n} \cdot \boldsymbol{\sigma} \cdot \boldsymbol{n}) \mathrm{d} S_{n}
$$

in terms of $\boldsymbol{X}=\left(\boldsymbol{U}^{(1)}, \ldots, \boldsymbol{U}^{(N)}\right)$ and $\left(\boldsymbol{u}_{\infty}, \boldsymbol{\sigma}_{\infty}, \gamma_{1}, \ldots, \gamma_{N}\right)$. As seen below, $\boldsymbol{I}_{i}^{(n)}$ arises when enforcing (2.5). Invoking the far-field expansion of the Stokes equations (Lamb 1932) we first observe that $|\boldsymbol{u}|$ and $\left|\boldsymbol{u}_{i}^{(n)}\right|$ decay at least as fast as $1 / r$ whereas $p, p_{i}^{(n)},|\boldsymbol{\sigma} \cdot \boldsymbol{x} / r|$ and $\left|\boldsymbol{\sigma}_{i}^{(n)} \cdot \boldsymbol{x} / r\right|$ decay at least as fast as $1 / r^{2}$ far from the cluster. Thus, the usual reciprocal identity (Happel \& Brenner 1973) for the Stokes flows 
$(\boldsymbol{u}, p)$, and $\left(\boldsymbol{u}_{i}^{(n)}, p_{i}^{(n)}\right)$ is

$$
\int_{S} \boldsymbol{u} \cdot \boldsymbol{\sigma}_{i}^{(n)} \cdot \boldsymbol{n} \mathrm{d} S=\int_{S} \boldsymbol{u}_{i}^{(n)} \cdot \boldsymbol{\sigma} \cdot \boldsymbol{n} \mathrm{d} S, \quad S=\cup_{m=1}^{N} S_{m}
$$

Since $\boldsymbol{\sigma}_{i}^{(n)} \cdot \boldsymbol{n}$ is normal on each surface $S_{m}$ it immediately follows that

$$
\int_{S} \boldsymbol{u} \cdot \boldsymbol{\sigma}_{i}^{(n)} \cdot \boldsymbol{n} \mathrm{d} S=\int_{S}(\boldsymbol{u} \cdot \boldsymbol{n})\left(\boldsymbol{n} \cdot \boldsymbol{\sigma}_{i}^{(n)} \cdot \boldsymbol{n}\right) \mathrm{d} S .
$$

Exploiting on $S_{m}$ the boundary condition $\boldsymbol{u}_{i}^{(n)} \cdot \boldsymbol{n}=\delta_{n m} \boldsymbol{e}_{i} \cdot \boldsymbol{n}$, one also easily obtains

$$
\begin{aligned}
\int_{S} \boldsymbol{u}_{i}^{(n)} \cdot \boldsymbol{\sigma} \cdot \boldsymbol{n} \mathrm{d} S & =\int_{S}\left\{\left(\boldsymbol{u}_{i}^{(n)} \cdot \boldsymbol{n}\right)(\boldsymbol{n} \cdot \boldsymbol{\sigma} \cdot \boldsymbol{n})+\boldsymbol{u}_{i}^{(n)} \cdot[\boldsymbol{\sigma} \cdot \boldsymbol{n}-(\boldsymbol{n} \cdot \boldsymbol{\sigma} \cdot \boldsymbol{n}) \boldsymbol{n}]\right\} \mathrm{d} S \\
& =\int_{S_{n}}\left(\boldsymbol{e}_{i} \cdot \boldsymbol{n}\right)(\boldsymbol{n} \cdot \boldsymbol{\sigma} \cdot \boldsymbol{n}) \mathrm{d} S_{n}+\int_{S} \boldsymbol{u}_{i}^{(n)} \cdot[\boldsymbol{\sigma} \cdot \boldsymbol{n}-(\boldsymbol{n} \cdot \boldsymbol{\sigma} \cdot \boldsymbol{n}) \boldsymbol{n}] \mathrm{d} S
\end{aligned}
$$

By virtue of (2.10)-(2.11), our equality (2.9) thus yields

$$
I_{i}^{(n)}=\sum_{m=1}^{N} \int_{S_{m}}\left\{(\boldsymbol{u} \cdot \boldsymbol{n})\left(\boldsymbol{n} \cdot \boldsymbol{\sigma}_{i}^{(n)} \cdot \boldsymbol{n}\right)-\boldsymbol{u}_{i}^{(n)} \cdot[\boldsymbol{\sigma} \cdot \boldsymbol{n}-(\boldsymbol{n} \cdot \boldsymbol{\sigma} \cdot \boldsymbol{n}) \boldsymbol{n}]\right\} \mathrm{d} S_{m} .
$$

Furthermore, note that under the conditions (2.5) we also impose

$$
-\boldsymbol{F}^{(n)} \cdot \boldsymbol{e}_{i}=\int_{S_{n}} \boldsymbol{e}_{i} \cdot \boldsymbol{\sigma} \cdot \boldsymbol{n} \mathrm{d} S_{n}=I_{i}^{(n)}+\int_{S_{n}} \boldsymbol{e}_{i} \cdot[\boldsymbol{\sigma} \cdot \boldsymbol{n}-(\boldsymbol{n} \cdot \boldsymbol{\sigma} \cdot \boldsymbol{n}) \boldsymbol{n}] \mathrm{d} S_{n} .
$$

Substituting (2.12) into (2.13) and taking into account the boundary conditions (2.3) for the perturbation flow $(\boldsymbol{u}, p)$, one finally arrives at the following key linear system for the $3 N$ unknown Cartesian velocity components $U_{j}^{(m)}=\boldsymbol{U}^{(m)} \cdot \boldsymbol{e}_{j}$ :

$$
\sum_{m=1}^{N} \sum_{j=1}^{3} A_{i j}^{(n),(m)} U_{j}^{(m)}=C_{i}^{(n)}+D_{i}^{(n)}=B_{i}^{(n)}, \quad i \in\{1,2,3\} \quad \text { and } \quad n=\in\{1, \ldots, N\}
$$

with the definitions

$$
\begin{aligned}
& A_{i j}^{(n),(m)}=\int_{S_{m}}\left(\boldsymbol{e}_{j} \cdot \boldsymbol{n}\right)\left(\boldsymbol{n} \cdot \boldsymbol{\sigma}_{i}^{(n)} \cdot \boldsymbol{n}\right) \mathrm{d} S_{m}, \quad C_{i}^{(n)}=\sum_{m=1}^{N} \int_{S_{m}}\left(\boldsymbol{u}_{\infty} \cdot \boldsymbol{n}\right)\left(\boldsymbol{n} \cdot \boldsymbol{\sigma}_{i}^{(n)} \cdot \boldsymbol{n}\right) \mathrm{d} S_{m}, \\
& D_{i}^{(n)}=-\boldsymbol{F}^{(n)} \cdot \boldsymbol{e}_{i}-\sum_{m=1}^{N} \int_{S_{m}}\left(\boldsymbol{u}_{i}^{(n)}-\delta_{n m} \boldsymbol{e}_{i}\right) \cdot\left[\nabla_{s} \gamma_{m}+\boldsymbol{\sigma}_{\infty} \cdot \boldsymbol{n}-\left(\boldsymbol{n} \cdot \boldsymbol{\sigma}_{\infty} \cdot \boldsymbol{n}\right) \boldsymbol{n}\right] \mathrm{d} S_{m} .
\end{aligned}
$$

Adopting the standard summation convention and introducing the matrices $\boldsymbol{B}^{(m)}$ and $\boldsymbol{A}^{(n),(m)}$ associated with the vectors $B_{i}^{(m)} \boldsymbol{e}_{i}$ and the second-rank tensors $A_{i j}^{(n),(m)} \boldsymbol{e}_{i} \otimes \boldsymbol{e}_{j}$ respectively, (2.14) readily admits the condensed notation

$$
\left(\begin{array}{ccccc}
\boldsymbol{A}^{(1),(1)} & \ldots & \boldsymbol{A}^{(1),(m)} & \ldots & \boldsymbol{A}^{(1),(N)} \\
\ldots & \ldots & \ldots & \ldots & \ldots \\
\boldsymbol{A}^{(n),(1)} & \ldots & \boldsymbol{A}^{(n),(m)} & \ldots & \boldsymbol{A}^{(n),(N)} \\
\ldots & \ldots & \ldots . & \ldots & \ldots \\
\boldsymbol{A}^{(N),(1)} & \ldots & \boldsymbol{A}^{(N),(m)} & \ldots & \boldsymbol{A}^{(N),(N)}
\end{array}\right)\left(\begin{array}{c}
\boldsymbol{U}^{(1)} \\
\ldots \\
\boldsymbol{U}^{(m)} \\
\ldots \\
\boldsymbol{U}^{(N)}
\end{array}\right)=\left(\begin{array}{c}
\boldsymbol{B}^{(1)} \\
\ldots \\
\boldsymbol{B}^{(n)} \\
\ldots \\
\boldsymbol{B}^{(N)}
\end{array}\right) .
$$


Let us now replace the perturbation flow $(\boldsymbol{u}, p)$ by $\left(\boldsymbol{u}_{j}^{(m)}, p_{j}^{(m)}\right)$ with stress tensor $\boldsymbol{\sigma}_{j}^{(m)}$ in our relations (2.9) and (2.11). Since $\boldsymbol{\sigma}_{j}^{(m)} \cdot \boldsymbol{n}$ is normal to $S$, it follows that

$$
\int_{S} \boldsymbol{u}_{j}^{(m)} \cdot \boldsymbol{\sigma}_{i}^{(n)} \cdot \boldsymbol{n} \mathrm{d} S=\int_{S} \boldsymbol{u}_{i}^{(n)} \cdot \boldsymbol{\sigma}_{j}^{(m)} \cdot \boldsymbol{n} \mathrm{d} S=\int_{S_{n}}\left(\boldsymbol{e}_{i} \cdot \boldsymbol{n}\right)\left(\boldsymbol{n} \cdot \boldsymbol{\sigma}_{j}^{(m)} \cdot \boldsymbol{n}\right) \mathrm{d} S_{n}=A_{j i}^{(m),(n)} .
$$

Switching the index pairs $(n, i)$ and $(m, j)$ in (2.18) then yields $\boldsymbol{A}_{i j}^{(n),(m)}=A_{j i}^{(m),(n)}$. The $3 N \times 3 N$ matrix $\boldsymbol{A}$ arising in (2.17) is thus symmetric. Moreover, the rate $E$ of dissipation of mechanical energy in any steady Stokes flow $\left(\boldsymbol{u}^{\prime}, p^{\prime}\right)$, with stress tensor $\sigma^{\prime}$, zero body force and quiessence far from the cluster, obeys (Happel \& Brenner 1973)

$$
-E=\int_{S} \boldsymbol{u}^{\prime} \cdot \boldsymbol{\sigma}^{\prime} \cdot \boldsymbol{n} \mathrm{d} S=\sum_{l=1}^{N} \int_{S_{l}} \boldsymbol{u}^{\prime} \cdot \boldsymbol{\sigma}^{\prime} \cdot \boldsymbol{n} \mathrm{d} S_{l}<0 .
$$

Accordingly, for any given velocity $\boldsymbol{X}=\left(\boldsymbol{U}^{(1)}, \ldots, \boldsymbol{U}^{(N)}\right)$, if we select $\left(\boldsymbol{u}^{\prime}, p^{\prime}\right)$ and $\boldsymbol{\sigma}^{\prime}$ as

$$
\boldsymbol{u}^{\prime}=\sum_{m=1}^{N} \sum_{j=1}^{3} U_{j}^{(m)} \boldsymbol{u}_{j}^{(m)}, \quad p^{\prime}=\sum_{m=1}^{N} \sum_{j=1}^{3} U_{j}^{(m)} p_{j}^{(m)}, \quad \boldsymbol{\sigma}^{\prime}=\sum_{n=1}^{N} \sum_{i=1}^{3} U_{i}^{(n)} \boldsymbol{\sigma}_{i}^{(n)}
$$

and recall that $\boldsymbol{\sigma}_{i}^{(n)} \cdot \boldsymbol{n} \wedge \boldsymbol{n}=\mathbf{0}$ we obtain (with a summation over indices $n, m, i$ and $j$ )

$$
\int_{S} \boldsymbol{u}^{\prime} \cdot \boldsymbol{\sigma}^{\prime} \cdot \boldsymbol{n} \mathrm{d} S=\sum_{l=1}^{N}\left[\int_{S_{l}}\left(\boldsymbol{u}_{j}^{(m)} \cdot \boldsymbol{n}\right)\left(\boldsymbol{n} \cdot \boldsymbol{\sigma}_{i}^{(n)} \cdot \boldsymbol{n}\right) \mathrm{d} S_{l}\right] U_{j}^{(m)} U_{i}^{(n)}=A_{i j}^{(n),(m)} U_{j}^{(m)} U_{i}^{(n)}<0 .
$$

The real-valued matrix $\boldsymbol{A}$ is thus both symmetric and negative-definite. The linear equations (2.14) therefore admit a unique solution $\boldsymbol{X}$ for arbitrarily prescribed capillary effects $\left(\gamma_{1}, \ldots, \gamma_{N}\right)$, net forces $\boldsymbol{F}^{(n)}$ and non-uniform ambient Stokes flow $\left(\boldsymbol{u}_{\infty}, p_{\infty}\right)$. This establishes the well posedness of the advocated approach. The main result of the present paper is that, as previously mentioned and evidenced by our definitions (2.15)-(2.16), one can determine the velocities $\boldsymbol{U}^{(n)}$ by evaluating a very few quantities defined on the surfaces $S_{m}$ : the tangential velocities $\boldsymbol{u}_{i}^{(n)}-\delta_{n m} \boldsymbol{e}_{i}$ and the normal stresses $\boldsymbol{\sigma}_{i}^{(n)} \cdot \boldsymbol{n}$ induced by the Stokes flows $\left(\boldsymbol{u}_{i}^{(n)}, p_{i}^{(n)}\right)$. As will be established in a future numerical implementation (Sellier 2004), these vectors obey coupled boundary-integral equations on $S$, and one thus only needs to mesh the surface of each bubble rather than the unbounded fluid domain.

\subsection{Case of a single bubble}

Whilst it requires a numerical treatment for $N \geqslant 2$, the advocated strategy reduces to a straightforward analytical application for a single spherical bubble $\mathscr{P}_{1}$, with centre $O_{1}=O$, radius $a_{1}=a$, surface $S_{1}$ and surface tension $\gamma_{1}=\gamma$. By virtue of (2.14)-(2.16), the net hydrodynamic force $\boldsymbol{F}=-\boldsymbol{F}^{(1)}$ and the velocity $\boldsymbol{U}$ experienced by $\mathscr{P}_{1}$ obey

$$
\boldsymbol{F} \cdot \boldsymbol{e}_{i}=\int_{S_{1}}\left\{\left(\boldsymbol{u}_{i}^{(1)}-\boldsymbol{e}_{i}\right) \cdot\left[\nabla_{s} \gamma+\boldsymbol{\sigma}_{\infty} \cdot \boldsymbol{n}-\left(\boldsymbol{n} \cdot \boldsymbol{\sigma}_{\infty} \cdot \boldsymbol{n}\right) \boldsymbol{n}\right]+\left(\boldsymbol{n} \cdot \boldsymbol{\sigma}_{i}^{(1)} \cdot \boldsymbol{n}\right)\left(\boldsymbol{U}-\boldsymbol{u}_{\infty}\right) \cdot \boldsymbol{n}\right\} \mathrm{d} S_{1}
$$


with $\left(\boldsymbol{u}_{i}^{(1)}, p_{i}^{(1)}\right)$ of stress tensor $\boldsymbol{\sigma}_{i}^{(1)}$ subject to (2.6)-(2.7). As detailed in Appendix B one obtains on the surface $S_{1}$

$$
\boldsymbol{u}_{i}^{(1)}-\boldsymbol{e}_{i}=\left[\left(\boldsymbol{e}_{i} \cdot \boldsymbol{n}\right) \boldsymbol{n}-\boldsymbol{e}_{i}\right] / 2, \quad \boldsymbol{\sigma}_{i}^{(1)} \cdot \boldsymbol{n}=-3 \mu\left(\boldsymbol{e}_{i} \cdot \boldsymbol{n}\right) \boldsymbol{n} / a
$$

which will be of interest as a future numerical benchmark. After some algebra, also displayed in Appendix B, (2.22) thereafter gives

$$
\boldsymbol{F}=-4 \pi \mu a\left[\boldsymbol{U}-\boldsymbol{u}_{\infty}(O)\right]-\frac{1}{2} \int_{S_{1}} \nabla_{s} \gamma \mathrm{d} S_{1}
$$

with $\boldsymbol{u}_{\infty}(O)$ denoting the velocity of the ambient flow $\boldsymbol{u}_{\infty}$ at the bubble centre $O$. Note that (2.24) agrees well (as special cases) with results established for a single drop in the absence of capillary effects or ambient flow by Hetsroni \& Haber (1970) or Subramanian (1985). However, Hetsroni \& Haber (1970) resort to a more involved treatment and Subramanian (1985) appeals to other auxiliary Stokes flows $\left(\boldsymbol{u}_{i}^{(1)}, p_{i}^{(1)}\right)$.

\section{Application to the thermocapillary migration of freely suspended and spherical bubbles}

This section applies the proposed procedure to the thermocapillary motion of spherical bubbles freely immersed in a quiescent liquid.

\subsection{General case}

Each bubble $\mathscr{P}_{n}$ is freely suspended $\left(\boldsymbol{F}^{(n)}=\mathbf{0}\right)$, of surface tension $\gamma_{n}$ depending solely on the temperature $T$ with $\mathrm{d} \gamma_{n} / \mathrm{d} T=\gamma_{n}^{\prime}$ constant and, for common substances, negative. The $N$-bubble cluster is embedded in a quiescent liquid $\left(\boldsymbol{u}_{\infty}=\mathbf{0}, p_{\infty}=0\right)$ and a divergence-free but not necessarily uniform temperature gradient $\nabla T_{\infty}$. The bubbles modify $T_{\infty}$ and we denote the disturbed temperature by $T_{\infty}+T^{\prime}$. Under these assumptions and the standard summation convention, (2.14)-(2.16) readily become

$$
\sum_{m=1}^{N} \sum_{j=1}^{3} A_{i j}^{(n),(m)} U_{j}^{(m)}=\sum_{m=1}^{N} \int_{S_{m}} \gamma_{m}^{\prime}\left(\delta_{n m} \boldsymbol{e}_{i}-\boldsymbol{u}_{i}^{(n)}\right) \cdot \nabla_{s}\left[T_{\infty}+T^{\prime}\right] \mathrm{d} S_{m} .
$$

Neglecting the thermal diffusivity of the gas inside the bubbles with respect to the constant liquid thermal diffusivity $\alpha>0$ and assuming that the Marangoni number $M a=U a / \alpha$ is negligible, $T^{\prime}$ obeys the well-posed exterior Neumann problem

$$
\begin{aligned}
\nabla^{2} T^{\prime} & =0 \text { in } \Omega, \nabla T^{\prime} \rightarrow \mathbf{0} \quad \text { as } r \rightarrow \infty \text { and } \nabla T^{\prime} \cdot \boldsymbol{n} \\
& =-\nabla T_{\infty} \cdot \boldsymbol{n} \text { on } S=\cup_{m=1}^{N} S_{m} .
\end{aligned}
$$

As will be shown in Part 2 (Sellier 2004), (3.2) makes it possible to compute on each surface $S_{m}$ the required vector $\nabla_{s} T^{\prime}$ by solving a boundary-integral equation on the entire boundary $S$. Recalling our similar remarks for the surface quantities $\boldsymbol{u}_{i}^{(n)}-\delta_{n m} \boldsymbol{e}_{i}$ and $\boldsymbol{\sigma}_{i}^{(n)} \cdot \boldsymbol{n}$, the numerical determination of the velocities $\boldsymbol{U}^{(n)}$ by means of (2.6)-(2.7) and (3.1)-(3.2) will require only the meshing of $S$.

\subsection{Case of equivalent bubbles}

The bubbles are equivalent whenever $a_{1} \gamma_{1}^{\prime}=\cdots=a_{N} \gamma_{N}^{\prime}=K>0$. In practice, such circumstances might occur only for clusters consisting of identical bubbles $\left(a_{1}=\right.$ $\cdots=a_{N}$ and $\gamma_{1}^{\prime}=\cdots=\gamma_{N}^{\prime}$ ). As nicely proved in Wang et al. (1994), equivalent 
bubbles immersed in a uniform temperature gradient $\nabla T_{\infty}$ adopt the same velocities

$$
\boldsymbol{U}^{(1)}=\cdots=\boldsymbol{U}^{(N)}=-\frac{K}{2 \mu} \nabla T_{\infty}
$$

Unfortunately, it is not straightforward to analytically deduce (3.3) from (3.1)-(3.2) when bubbles are equivalent and $\nabla T_{\infty}$ is uniform. Another formulation is needed and the crucial step consists of noting that $\left(\boldsymbol{u}^{\prime}, p^{\prime}\right)=\left(\nabla T^{\prime}, 0\right)$, with stress tensor $\boldsymbol{\sigma}^{\prime}$, is a steady Stokes flow quiescent at infinity. Thus, one can replace $(\boldsymbol{u}, p)$ with $\left(\boldsymbol{u}^{\prime}, p^{\prime}\right)$ in (2.9)-(2.11). Setting $\boldsymbol{\sigma}_{t}^{\prime}=\boldsymbol{\sigma}^{\prime} \cdot \boldsymbol{n}-\left(\boldsymbol{n} \cdot \boldsymbol{\sigma}^{\prime} \cdot \boldsymbol{n}\right) \boldsymbol{n}$ on $S$, it follows that

$$
\int_{S}\left(\boldsymbol{u}^{\prime} \cdot \boldsymbol{n}\right)\left(\boldsymbol{n} \cdot \boldsymbol{\sigma}_{i}^{(n)} \cdot \boldsymbol{n}\right) \mathrm{d} S=\int_{S_{n}}\left(\boldsymbol{e}_{i} \cdot \boldsymbol{n}\right)\left(\boldsymbol{n} \cdot \boldsymbol{\sigma}^{\prime} \cdot \boldsymbol{n}\right) \mathrm{d} S_{n}+\int_{S} \boldsymbol{u}_{i}^{(n)} \cdot \boldsymbol{\sigma}_{t}^{\prime} \mathrm{d} S .
$$

Furthermore, as established in Appendix C,

$$
\int_{S_{n}} \boldsymbol{e}_{i} \cdot \boldsymbol{\sigma}^{\prime} \cdot \boldsymbol{n} \mathrm{d} S_{n}=\mathbf{0}, \quad \frac{\boldsymbol{\sigma}_{t}^{\prime}}{2 \mu}=\nabla_{s}\left[\nabla T^{\prime} \cdot \boldsymbol{n}\right]-\frac{\nabla_{s} T^{\prime}}{a_{n}} \quad \text { on } \quad S_{n} .
$$

Accordingly, exploiting the boundary condition (3.2) the expression (3.4) becomes

$$
\sum_{m=1}^{N} \int_{S_{m}}\left\{\left(\frac{\nabla T_{\infty} \cdot \boldsymbol{n}}{2 \mu}\right)\left(\boldsymbol{n} \cdot \boldsymbol{\sigma}_{i}^{(n)} \cdot \boldsymbol{n}\right)-\left(\boldsymbol{u}_{i}^{(n)}-\delta_{n m} \boldsymbol{e}_{i}\right) \cdot\left\{\nabla_{s}\left[\nabla T_{\infty} \cdot \boldsymbol{n}\right]+\frac{\nabla_{s} T^{\prime}}{a_{m}}\right\}\right\} \mathrm{d} S_{m}=0
$$

For equivalent bubbles with $a_{n} \gamma_{n}=K>0$ the combination of (3.1) and (3.6) thus yields

$$
\begin{array}{r}
\sum_{m=1}^{N}\left[\int_{S_{m}}\left(\boldsymbol{n} \cdot \boldsymbol{\sigma}_{i}^{(n)} \cdot \boldsymbol{n}\right) \boldsymbol{n} \mathrm{d} S_{m}\right] \cdot \boldsymbol{U}^{(m)}=-\frac{K}{2 \mu} \sum_{m=1}^{N} \int_{S_{m}}\left(\boldsymbol{n} \cdot \boldsymbol{\sigma}_{i}^{(n)} \cdot \boldsymbol{n}\right)\left(\boldsymbol{n} \cdot \nabla T_{\infty}\right) \mathrm{d} S_{m} \\
+K \sum_{m=1}^{N} \int_{S_{m}}\left(\boldsymbol{u}_{i}^{(n)}-\delta_{n m} \boldsymbol{e}_{i}\right) \cdot\left\{\nabla_{s}\left[\nabla T_{\infty} \cdot \boldsymbol{n}\right]-\frac{\nabla_{s} T_{\infty}}{a_{m}}\right\} \mathrm{d} S_{m} .
\end{array}
$$

Clearly, for equivalent bubbles one may solve (3.7) instead of (3.1)-(3.2) and therefore does not need to determine the disturbance temperature $T^{\prime}$. This property holds for any divergence-free temperature gradient $\nabla T_{\infty}$. When exploiting (3.7) two cases arise.

(i) $N=1$. In this case we consider a single bubble $\mathscr{P}_{1}$ with radius $a$, centre $O_{1}=O$, boundary $S_{1}$ and surface tension $\gamma$ with $K=\gamma^{\prime} a$. Noting that $\boldsymbol{n} \cdot \boldsymbol{\sigma}_{i}^{(1)} \cdot \boldsymbol{n}=$ $-3 \mu\left(\boldsymbol{e}_{i} \cdot \boldsymbol{n}\right) / a$ (recall (2.23)) and replacing the Stokes flow $\left(\boldsymbol{u}_{\infty}, p_{\infty}\right)$ in (B 5) and (B 7) by the trivial Stokes flows $\left(\boldsymbol{e}_{j}, 0\right)$ and $\left(\nabla T_{\infty}, 0\right)$, one easily obtains

$$
\int_{S_{1}} \frac{\left(\boldsymbol{n} \cdot \boldsymbol{\sigma}_{i}^{(1)} \cdot \boldsymbol{n}\right)\left(\boldsymbol{n} \cdot \boldsymbol{e}_{j}\right)}{4 \pi \mu a} \mathrm{~d} S_{1}=-\delta_{i j}, \quad \int_{S_{1}} \frac{\left(\boldsymbol{n} \cdot \boldsymbol{\sigma}_{i}^{(1)} \cdot \boldsymbol{n}\right)\left(\boldsymbol{n} \cdot \nabla T_{\infty}\right)}{4 \pi \mu a} \mathrm{~d} S_{1}=-\nabla T_{\infty}(O) \cdot \boldsymbol{e}_{i}
$$

where $\nabla T_{\infty}(0)$ denotes the ambient temperature gradient at the bubble centre $O_{1}=O$. Morevover, as shown in Appendix C,

$$
\mathscr{L}=2 \int_{S_{1}}\left(\boldsymbol{u}_{i}^{(1)}-\boldsymbol{e}_{i}\right) \cdot\left\{\nabla_{s}\left(\nabla T_{\infty} \cdot \boldsymbol{n}\right)-\frac{\nabla_{s} T_{\infty}}{a}\right\} \mathrm{d} S_{1}=0 .
$$

Substituting (3.8) and (3.9) into (3.7) the velocity $\boldsymbol{U}$ of the single bubble becomes $\boldsymbol{U}=-\gamma^{\prime} a \nabla T_{\infty}(0) /(2 \mu)$, a result that agrees with Subramanian (1985). If $\nabla T_{\infty}$ is uniform one of course obtains the solution (1.1). 
(ii) $N \geqslant 2$. In this case we consider at least two equivalent bubbles. If $\nabla T_{\infty}$ is uniform, we note that $\nabla_{s}\left(\nabla T_{\infty}\right)-\nabla_{s} T_{\infty} / a_{m}$ vanishes on $S_{m}$ (using (A 3)) and, recalling the definition (2.15) of $\boldsymbol{A}_{i j}^{(n),(m)}$, (3.7) readily becomes

$$
\sum_{m=1}^{N} \sum_{j=1}^{3} A_{i j}^{(n),(m)}\left[U_{j}^{(m)}+\frac{K}{2 \mu} \nabla T_{\infty} \cdot \boldsymbol{e}_{j}\right]=0 .
$$

Hence, one immediately recovers (3.3). Whenever $\nabla T_{\infty}$ is non-uniform only a numerical treatment of (3.7) is possible for $N \geqslant 2$. Such a task is the subject of Part 2.

\section{Conclusions}

A new approach has been proposed to determine the motion of torque-free spherical bubbles immersed in a non-uniform ambient Stokes flow and subject to arbitrary capillary effects and net forces. As established, the procedure is well-posed. In addition and unlike previous studies, it only requires the evaluation of a few quantities on the surface of each bubble and therefore circumvents the computation of other quantities (such as the liquid flow) in the unbounded fluid domain. The resulting formulation has been given for the thermocapillary migration of non-conducting and freely suspended bubbles and it has been found that, for any harmonic ambient temperature, it is unnecessary to evaluate the disturbance temperature when bubbles are equivalent. Furthermore, all the analytical results easily obtained for a single bubble or the thermocapillary motion of equivalent bubbles subject to a uniform temperature gradient agree well with previous works.

In Part 2 of this work (Sellier 2004), a suitable numerical implementation will be presented, and it will be shown how to obtain all the required surface quantities by solving relevant boundary-integral equations on the entire boundary $S=\cup_{m=1}^{N} S_{m}$. This property will then permit us to investigate the thermocapillary motion of fully three-dimensional clusters consisting of at least three non-equivalent bubbles.

\section{Appendix A. Definition of surface operator $\nabla_{s}$ and proof of (2.4)}

For a prescribed unit vector $\boldsymbol{e}$, we define a point $M$ on $S_{n}$ by its spherical angles $(\theta, \varphi)$ with $\boldsymbol{O}_{n} \boldsymbol{M} \cdot \boldsymbol{e}=a_{n} \cos \theta, \theta \in[0, \pi]$ and $\varphi \in[0,2 \pi]$. Setting $\boldsymbol{e}_{\varphi}=\boldsymbol{e} \wedge \boldsymbol{O}_{n} \boldsymbol{M} /$ $\left[a_{n} \sin \theta\right]$ and $\boldsymbol{e}_{\theta}=\boldsymbol{e}_{\varphi} \wedge \boldsymbol{O}_{n} \boldsymbol{M} / a_{n}$, one obtains $\mathbf{d} \boldsymbol{M}=a_{n}\left[\mathrm{~d} \theta \boldsymbol{e}_{\theta}+\sin \theta \mathrm{d} \varphi \boldsymbol{e}_{\varphi}\right]$ on $S_{n}$. The surface gradient $\nabla_{s} b$ of a smooth function $b(\theta, \varphi)$ obeys $\mathrm{d} b=\nabla_{s} b \cdot \mathbf{d} \boldsymbol{M}$ and thus is written

$$
\nabla_{s} b=\frac{1}{a_{n}}\left\{\frac{1}{\sin \theta} \frac{\partial b}{\partial \varphi} \boldsymbol{e}_{\varphi}+\frac{\partial b}{\partial \theta} \boldsymbol{e}_{\theta}\right\}, \boldsymbol{e}_{\varphi}=\boldsymbol{e} \wedge \boldsymbol{O}_{n} \boldsymbol{M} /\left[a_{n} \sin \theta\right], \boldsymbol{e}_{\theta}=\boldsymbol{e}_{\varphi} \wedge \boldsymbol{O}_{n} \boldsymbol{M} / a_{n} .
$$

From $\partial \boldsymbol{e}_{\varphi} / \partial \theta=\mathbf{0}, \partial \boldsymbol{e}_{\theta} / \partial \varphi=\cos \theta \boldsymbol{e}_{\varphi}$ and $\mathrm{d} S_{n}=a_{n}^{2} \sin \theta \mathrm{d} \theta \mathrm{d} \varphi$, (2.4) follows because

$$
\int_{S_{n}} \nabla_{s} b \wedge \boldsymbol{O}_{n} \boldsymbol{M} \mathrm{d} S_{n}=a_{n}^{2} \int_{0}^{\pi} \int_{0}^{2 \pi}\left[\frac{\partial\left(b \boldsymbol{e}_{\theta}\right)}{\partial \varphi}-\frac{\partial\left(b \sin \theta \boldsymbol{e}_{\varphi}\right)}{\partial \theta}\right] \mathrm{d} \varphi \mathrm{d} \theta=\mathbf{0} .
$$

On $S_{n}$ we note that $\boldsymbol{n}=\boldsymbol{O}_{n} \boldsymbol{M} /\left|\boldsymbol{O}_{n} \boldsymbol{M}\right|=\boldsymbol{n}(\theta, \varphi), \partial \boldsymbol{n} / \partial \theta=\boldsymbol{e}_{\theta}$ and $\partial \boldsymbol{n} / \partial \varphi=\sin \theta \boldsymbol{e}_{\varphi}$. Exploiting (A 1) we thus have, for any constant vector $\boldsymbol{v}$ and any function $\mathrm{d}\left(\left|\boldsymbol{O}_{n} \boldsymbol{M}\right|, \theta, \varphi\right)$,

$$
a_{n} \nabla_{s}[b(\boldsymbol{n} \cdot \boldsymbol{v})]=a_{n}(\boldsymbol{n} \cdot \boldsymbol{v}) \nabla_{s} b+b[\boldsymbol{v}-(\boldsymbol{n} \cdot \boldsymbol{v}) \boldsymbol{n}], \quad \nabla_{s} d=\nabla d-(\nabla d \cdot \boldsymbol{n}) \boldsymbol{n} .
$$




\section{Appendix B. Establishing (2.23)-(2.24)}

From Hadamard (1911) and Rybczynski (1911) the flow $\left(\boldsymbol{u}_{i}^{(1)}, p_{i}^{(1)}\right)$, axisymmetric about the axis $\left(O, \boldsymbol{e}_{i}\right)$, has velocity of the form

$$
\boldsymbol{u}_{i}^{(1)}=\left(\frac{2 D}{r^{3}}+\frac{2 C}{r}\right) \cos \theta \boldsymbol{e}_{r}+\left(\frac{D}{r^{3}}-\frac{C}{r}\right) \sin \theta \boldsymbol{e}_{\theta} \text { for } r \geqslant a
$$

with $r=|\boldsymbol{O} M|, \boldsymbol{O} M \cdot \boldsymbol{e}_{i}=r \cos \theta, \boldsymbol{e}_{r}=\boldsymbol{O} M / r$ and $\boldsymbol{e}_{\theta}=\left(\boldsymbol{e}_{i} \wedge \boldsymbol{e}_{r}\right) \wedge \boldsymbol{e}_{r}$. Since $\nabla p_{i}^{(1)}=$ $\mu \nabla^{2} \boldsymbol{u}_{i}^{(1)}$ with $p_{i}^{(1)} \rightarrow 0$ at infinity one also obtains $p_{i}^{(1)}=2 \mu C \cos \theta / r^{2}$ and

$$
\boldsymbol{\sigma}_{i}^{(1)} \cdot \boldsymbol{e}_{r}=-\frac{6 \mu}{r^{2}}\left\{\left(C+\frac{2 D}{r^{2}}\right) \cos \theta \boldsymbol{e}_{r}+\frac{D \sin \theta}{r^{2}} \boldsymbol{e}_{\theta}\right\} \text { for } r \geqslant a .
$$

On the boundary $S_{1}(r=a)$ the conditions $\boldsymbol{u}_{i}^{(1)} \cdot \boldsymbol{n}=\boldsymbol{e}_{i} \cdot \boldsymbol{n}$ and $\boldsymbol{\sigma}_{i}^{(1)} \cdot \boldsymbol{n}$ aligned with $\boldsymbol{n}=\boldsymbol{e}_{r}$ thus yield $2 a^{2} C+2 D=a^{3}$ and $D=0$. Hence, one obtains (2.23). It follows that

$$
\int_{S_{1}}\left[\left(\boldsymbol{u}_{i}^{(1)}-\boldsymbol{e}_{i}\right) \cdot \nabla_{s} \gamma+\left(\boldsymbol{n} \cdot \boldsymbol{\sigma}_{i}^{(1)} \cdot \boldsymbol{n}\right) \boldsymbol{U} \cdot \boldsymbol{n}\right] \mathrm{d} S_{1}=-\frac{1}{2} \int_{S_{1}} \nabla_{s} \gamma \cdot \boldsymbol{e}_{i} \mathrm{~d} S_{1}-4 \pi \mu a U_{i} .
$$

Let us now look at the quantities $K_{i}$ and $L_{i}$ such that

$\frac{3 \mu K_{i}}{a^{2}}=-\int_{S_{1}}\left(\boldsymbol{n} \cdot \boldsymbol{\sigma}_{i}^{(1)} \cdot \boldsymbol{n}\right) \boldsymbol{u}_{\infty} \cdot \boldsymbol{n} \mathrm{d} S_{1}, \quad \frac{3 L_{i}}{2 a^{2}}=\int_{S_{1}}\left(\boldsymbol{e}_{i}-\boldsymbol{u}_{i}^{(1)}\right) \cdot\left[\boldsymbol{\sigma}_{\infty} \cdot \boldsymbol{n}-\left(\boldsymbol{n} \cdot \boldsymbol{\sigma}_{\infty} \cdot \boldsymbol{n}\right) \boldsymbol{n}\right] \mathrm{d} S_{1}$.

Recalling that $x_{i}=a \boldsymbol{n} \cdot \boldsymbol{e}_{i}=\boldsymbol{O} M \cdot \boldsymbol{e}_{i}$ on $S_{1}$ whereas $\nabla \cdot \boldsymbol{u}_{\infty}=0$ and $\boldsymbol{\nabla} \cdot \boldsymbol{\sigma}_{\infty}=\mathbf{0}$ in the vicinity of $\mathscr{P}_{1}$, we obtain from (2.23) and the divergence theorem

$$
\begin{aligned}
& K_{i}=a \int_{S_{1}}\left(\boldsymbol{e}_{i} \cdot \boldsymbol{n}\right) \boldsymbol{u}_{\infty} \cdot \boldsymbol{n} \mathrm{d} S_{1}=\int_{\mathscr{P}_{1}} \nabla \cdot\left[x_{i} \boldsymbol{u}_{\infty}\right] \mathrm{d} v=\left[\int_{\mathscr{P}_{1}} \boldsymbol{u}_{\infty} \mathrm{d} v\right] \cdot \boldsymbol{e}_{i}, \\
& 3 L_{i}=-a^{2} \int_{S_{1}}\left(\boldsymbol{e}_{i} \cdot \boldsymbol{n}\right)\left(\boldsymbol{n} \cdot \boldsymbol{\sigma}_{\infty} \cdot \boldsymbol{n}\right) \mathrm{d} S_{1}=-\int_{\mathscr{P}_{1}} \nabla \cdot\left[x_{i} \boldsymbol{\sigma}_{\infty} \cdot \boldsymbol{O} M\right] \mathrm{d} v .
\end{aligned}
$$

We denote by $\left(\boldsymbol{u}_{\infty}(O), \nabla p_{\infty}(O)\right)$ the value of $\left(\boldsymbol{u}_{\infty}, \nabla p_{\infty}\right)$ at the bubble centre $O$ and set $S_{r}=\{M,|\boldsymbol{O} M|=r\}$. Since $\left(\boldsymbol{u}_{\infty}, p_{\infty}\right)$ is a Stokes flow (see (27) in Hetsroni, Wacholder $\&$ Haber 1971) and $\nabla p_{\infty} \cdot \boldsymbol{e}_{i}$ is harmonic we have the useful relations

$$
\int_{S_{r}} \boldsymbol{u}_{\infty} \mathrm{d} S=4 \pi r^{2}\left[\boldsymbol{u}_{\infty}(O)+\frac{r^{2}}{6 \mu} \nabla p_{\infty}(O)\right], \quad \int_{S_{r}} \nabla p_{\infty} \cdot \boldsymbol{e}_{i} \mathrm{~d} S=4 \pi r^{2} \nabla p_{\infty}(O) \cdot \boldsymbol{e}_{i} .
$$

Accordingly, one obtains

$$
K_{i}=\int_{0}^{a}\left[\int_{S_{r}} \boldsymbol{u}_{\infty} \cdot \boldsymbol{e}_{i} \mathrm{~d} S\right] \mathrm{d} r=\frac{4 \pi a^{3}}{3}\left[\boldsymbol{u}_{\infty}(O)+\frac{a^{2}}{10 \mu} \nabla p_{\infty}(O)\right] \cdot \boldsymbol{e}_{i} .
$$

Using Cartesian coordinates and the definition of the stress tensor $\sigma_{\infty}$, the reader may easily check that $\nabla \cdot\left[x_{i} \boldsymbol{\sigma}_{\infty} \cdot \boldsymbol{O} M\right]=x_{i}\left(\nabla \cdot \boldsymbol{\sigma}_{\infty}\right) \cdot \boldsymbol{O} M+\boldsymbol{e}_{i} \cdot \boldsymbol{\sigma}_{\infty} \cdot \boldsymbol{O} M-3 p_{\infty} x_{i}$. We thus have

$3 L_{i}=-\int_{\mathscr{P}_{1}} x_{i}\left(\nabla \cdot \boldsymbol{\sigma}_{\infty}\right) \cdot \boldsymbol{O} M \mathrm{~d} v-\int_{0}^{a}\left[\int_{S_{r}} \boldsymbol{e}_{i} \cdot \boldsymbol{\sigma}_{\infty} \cdot \boldsymbol{n} \mathrm{d} S\right] r \mathrm{~d} r+3 \int_{0}^{a}\left[\int_{S_{r}} p_{\infty} \boldsymbol{e}_{i} \cdot \boldsymbol{n} \mathrm{d} S\right] r \mathrm{~d} r$. 
Since $\boldsymbol{\nabla} \cdot \boldsymbol{\sigma}_{\infty}=\mathbf{0}$, only the last integral in (B 9) is non-zero. Setting $\mathscr{P}_{r}=$ $\{M,|\boldsymbol{O} M|<r\}$ and invoking the divergence theorem and (B 7), it thus appears that

$$
\begin{aligned}
L_{i}=\int_{0}^{a}\left[\int_{\mathscr{P}_{r}} \nabla p_{\infty} \cdot \boldsymbol{e}_{i} \mathrm{~d} S\right] r \mathrm{~d} r & =\int_{0}^{a}\left[\int_{0}^{r}\left(\int_{S_{\rho}} \nabla p_{\infty} \cdot \boldsymbol{e}_{i} \mathrm{~d} S\right) \mathrm{d} \rho\right] r \mathrm{~d} r \\
& =\frac{4 \pi a^{5}}{15} \nabla p_{\infty}(O) \cdot \boldsymbol{e}_{i} .
\end{aligned}
$$

Under (B 3)-(B 4),(B 8) and (B 10) it is then easy to deduce (2.24) from (2.22).

\section{Appendix C. Derivation of (3.5) and (3.9)}

We set $g_{, i}=\partial g / \partial x_{i}$ and note that $\boldsymbol{\sigma}^{\prime} \cdot \boldsymbol{e}_{i}=2 \mu \nabla\left(T_{, i}^{\prime}\right)$ for $\boldsymbol{u}^{\prime}=\nabla T^{\prime}$ and $p^{\prime}=0$. Under the following single-layer representation:

$$
T^{\prime}(M)=\sum_{m=1}^{N} T_{m}^{\prime}(M) \quad \text { in } \quad \Omega \quad \text { with } \quad T_{m}^{\prime}(M)=\int_{S_{m}} q_{m}(P) \mathrm{d} S_{m} / P M
$$

and observing that $\boldsymbol{e}_{i} \cdot \boldsymbol{\sigma}^{\prime} \cdot \boldsymbol{n}=\left(\boldsymbol{\sigma}^{\prime} \cdot \boldsymbol{e}_{i}\right) \cdot \boldsymbol{n}$ for the symmetric tensor $\boldsymbol{\sigma}^{\prime}$, one obtains

$$
\mathscr{I}=\int_{S_{n}} \boldsymbol{e}_{i} \cdot \boldsymbol{\sigma}^{\prime} \cdot \boldsymbol{n} \mathrm{d} S_{n}=2 \mu\left\{I_{n}+\sum_{m \neq n} I_{m}\right\}, \quad I_{l}=\int_{S_{n}} \nabla\left(T_{l, i}^{\prime}\right) \cdot \boldsymbol{n} \mathrm{d} S_{n} .
$$

By virtue of (C1), $T_{m, i}^{\prime}$ is harmonic in $\mathscr{P}_{n}$ for $m \neq n$ and $T_{n, i}^{\prime}$ is harmonic in $\mathbb{R}^{3} \backslash \mathscr{P}_{n}$. If $S_{n}(\rho)=\left\{M,\left|\boldsymbol{O}_{n} \boldsymbol{M}\right|=\rho\right\}$ for $\rho>a_{n}$, the divergence theorem yields

$$
I_{n}=\int_{S_{n}(\rho)} \nabla\left(T_{n, i}^{\prime}\right) \cdot \frac{\boldsymbol{O}_{n} \boldsymbol{M}}{\left|\boldsymbol{O}_{n} \boldsymbol{M}\right|} \mathrm{d} S, I_{m}=\int_{\mathscr{P}_{n}} \nabla^{2}\left(T_{m, i}^{\prime}\right) \mathrm{d} v=0 \quad \text { for } \quad m \neq n .
$$

In addition, as $\rho=\left|\boldsymbol{O}_{n} \boldsymbol{M}\right| \rightarrow \infty, T_{n}^{\prime}$ and $\left|\nabla\left(T_{n, i}^{\prime}\right)\right|$ decay at least as $1 / \rho$ and $1 / \rho^{3}$ on $S_{n}(\rho)$, respectively. Accordingly, $I_{n}$ vanishes and, as stated in (3.5), $\mathscr{I}=0$. Using the summation convention with $\boldsymbol{n}=n_{j} \boldsymbol{e}_{j}$ and noting $g_{, i j}=\left(g_{, i}\right)_{, j}$, on $S_{n}$ one also arrives at

$$
\boldsymbol{\sigma}^{\prime} \cdot \boldsymbol{n}=2 \mu T_{, i j}^{\prime} n_{j} \boldsymbol{e}_{i}, \quad \boldsymbol{\sigma}_{t}^{\prime}=\boldsymbol{\sigma}^{\prime} \cdot \boldsymbol{n}-\left(\boldsymbol{n} \cdot \boldsymbol{\sigma}^{\prime} \cdot \boldsymbol{n}\right) \boldsymbol{n}=2 \mu\left\{T_{, i j}^{\prime} n_{j}+\left(n_{k} T_{, k j}^{\prime} n_{j}\right) n_{i}\right\} \boldsymbol{e}_{i} .
$$

Exploiting the relation (A 3) for $\boldsymbol{v}=\boldsymbol{e}_{i}$, the reader may also easily check that

$$
\nabla_{s}\left(\nabla T^{\prime} \cdot \boldsymbol{n}\right)=n_{i} \nabla_{s}\left(T_{, i}^{\prime}\right)+\frac{T_{, i}^{\prime}}{a_{n}}\left[\boldsymbol{e}_{i}-n_{i} \boldsymbol{n}\right]=n_{i}\left[T_{, i j}^{\prime}-\left(T_{, i k}^{\prime} n_{k}\right) n_{j}\right] \boldsymbol{e}_{j}+\frac{\nabla_{s} T^{\prime}}{a_{n}} .
$$

Since $T_{, i j}^{\prime}=T_{, j i}^{\prime}$, one thus deduces the relation (3.5) for $\sigma_{t}^{\prime} /(2 \mu)$ from (C 4)-(C 5). From our definition (3.9) of $\mathscr{L},(2.23)$ and (C 4), it follows that

$$
\mathscr{L}=\int_{S_{1}}\left[\left(\boldsymbol{e}_{i} \cdot \boldsymbol{n}\right) \boldsymbol{n}-\boldsymbol{e}_{i}\right] \cdot\left[T_{\infty, k j} n_{j} \boldsymbol{e}_{k}\right] \mathrm{d} S_{1} .
$$

Using the correspondence $n_{i}=x_{i} / a$ and the divergence theorem, one arrives at

$$
\mathscr{L}=\int_{S_{1}}\left[\frac{x_{i}}{a} \frac{x_{j}}{a} T_{\infty, k j}-T_{\infty, i k}\right] n_{k} \mathrm{~d} S_{1}=\int_{\mathscr{P}_{1}}\left(\frac{x_{i} x_{j}}{a^{2}} T_{\infty, k j}-T_{\infty, i k}\right)_{, k} \mathrm{~d} v .
$$


For $T_{\infty}$ harmonic in $\mathscr{P}_{1}\left(T_{\infty, k k}=0\right)$ and $S_{r}$ or $\mathscr{P}_{r}$ defined as in Appendix B, we thus have

$$
a^{2} \mathscr{L}=\int_{\mathscr{P}_{1}} x_{j} T_{\infty, i j} \mathrm{~d} v=\int_{0}^{a} r\left[\int_{S_{r}} T_{\infty, i j} n_{j} \mathrm{~d} S\right] \mathrm{d} r=\int_{0}^{a} r\left[\int_{\mathscr{P}_{r}}\left(T_{\infty, i j}\right)_{, j} \mathrm{~d} v\right] \mathrm{d} r=0 .
$$

\section{REFERENCES}

Acrivos, A., Jefrrey, D. F. \& Saville, D. A. 1990 Particle migration in suspensions by thermocapillary or electrophoretic motion. J. Fluid Mech. 212, 95-110.

ANDERSON, J. L. 1985 Droplets interactions in thermocapillary motion. Intl J. Multiphase Flow 11, 813-824.

Feuillebois, F. 1989 Thermocapillary migration of two equal bubbles parallel to their line of centers. J. Colloid Interface Sci. 131, 267-274.

HADAMARD, J. 1911 Mouvement permanet lent d'une sphère liquide et visqueuse dans un liquide visqueux. C. R. Acad. Sci., Paris 152, 1735-1738.

HAPPEL, J. \& BRENNER, H. 1973 Low Reynolds Number Hydrodynamics. Martinus Nijhoff.

Hetsroni, G. \& Haber, S. 1970 Flow in and around a droplet or bubble submerged in an unbound arbitrary velocity field. Rheol. Acta 9, 488-496.

Hetsroni, G., Wacholder, E. \& Haber, S. 1971 The hydrodynamic resistance of a fluid sphere submerged in Stokes flows. Z. Angew. Math. Mech. 15, 45-50.

KeH, H. J. \& Chen, S. H. 1990 The axisymmetric thermocapillary motion of two fluid droplets. Intl J. Multiphase Flow 16, 515-527.

KeH, H. J. \& Chen, L. S. 1992 Droplet interactions in axisymmetric thermocapillary motion. J. Colloid Interface Sci. 151, 1-16.

KeH, H. J. \& Chen, L. S. 1993 Droplet interactions in thermocapillary migration. J. Chem. Engng Sci. 48, 3565-3582.

Lamb, H. 1932 Hydrodynamics, 6th edn. Cambridge University Press.

Meyyappan, M. \& SubRamanian, R. S. 1984 The thermocapillary motion of two bubbles oriented arbitrarily relative to a thermal gradient. J. Colloid Interface Sci. 97, 291-294.

Meyyappan, M., Wilcox, W. R. \& Subramanian, R. S. 1983 The slow axisymmetric motion of two bubbles in a thermal gradient. J. Colloid Interface Sci. 94, 243-257.

RybCZYNSKI, D. 1911 Über die fortschreitende Bewegung einer Flüssigen Kugel in einem zähen Medium. Bull. Acad. Sci. Cracovie, 1, 40-46.

SATRAPE, J. V. 1992 Interactions and collisions of bubbles in thermocapillary motion. Phys. Fluids A 4, 1883-1900.

Sellier, A. 2004 On the capillary motion of arbitrary clusters of spherical bubbles. Part 2. Boundary formulation and numerical results. In preparation.

Subramanian, R. S. 1985 The Stokes force on a droplet in an unbounded fluid medium due to capillary effects. J. Fluid Mech. 153, 389-400.

Sun, R. \& Hu, W. 2002 The thermocapillary motions of two bubbles in microgravity environment. J. Colloid Interface Sci. 97, 375-381.

WANG, Y., MaUry, R. \& ACRIVos, A. 1994 Thermocapillary migration of a bidisperse suspension of bubbles. J. Fluid Mech. 261, 47-64.

Wei, H. \& Subramanian, R. S. 1993 Thermocapillary migration of a small chain of bubbles. Phys. Fluids A 5, 1583-1595.

Young, N. O., Goldstein, J. S. \& BLock, M. J. 1959 The motion of bubbles in a vertical temperature gradient. J. Fluid Mech. 6, 350-356. 